
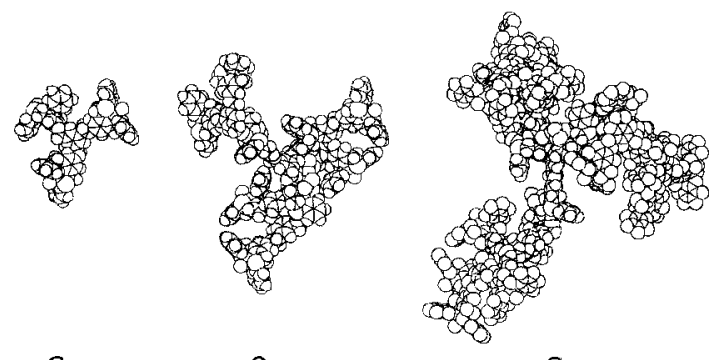

$\mathrm{G}_{0}$

$\mathrm{G}_{1}$

$\mathrm{G}_{2}$

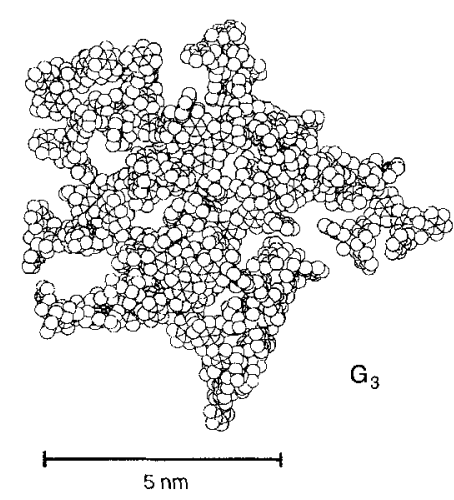

Abb. 2. Durch Kraftfeldrechnungen ermittelte Energieminimumstrukturen von $\mathbf{G}_{0}-\mathbf{G}_{3}$.

liert aufzubauen. Mit der beschriebenen Methode steht ein neues und vielseitiges Werkzeug zur Synthese von Dendrimeren zur Verfügung. Darüber hinaus sollte es nach diesem Konzept möglich sein, die Metallatome in den Generationen, die koordinierenden Liganden sowie die Struktur der Zentraleinheit zu variieren.

Eingegangen am 29. Dezember 1995 [Z 8690]

Stichworte: Dendrimere $\cdot$ Palladiumkomplexe $\cdot$ Supramolekulare Chemie

[1] Übersichtsartikel: G. Ozin, Adv. Mater. 1992, 15, 612-648.

[2] Zur Synthese eines Rezeptormoleküls mit einem nanometergroßen Hohlraum siehe: P. Timmerman, W. Verboom, F. C. J. M. van Veggel, W. P. van Hoorn, D. N. Reinhoudt, Angew. Chem. 1994, 106,1313-1316; Angew. Chem. Int Ed. Engl. 1994, 33, $1292-1295$.

[3] J. P. Mathias, E. E. Simanek, G. M. Whitesides, J. Am. Chem. Soc. 1994, 116, $4326 \cdot 4340$.

[4] N. Khazanovich. J. R. Granja, D. E. McRee, R. A. Milligan, M. R. Ghadiri, J. An. Chem. Soc. 1994, 116, 6011-6012.

[5] J.-M. Lehn, Supramolecular Chemistry, VCH, Weinheim, 1995, S. 139-197, zit. Lit.

[6] D. M. Rudkevich, W. Verboom, Z. Brzøzka, M. J. Palys, W. P. R. V. Stauthamer, G. J, van Hummel, S. M. Franken. S. Harkema, J. F. J. Engbersen, D. N. Reinhoudt. J. Am. Chem. Soc 1994, 116, 4341-4351.

[7] D. A. Tomalia, A. M. Naylor, W. A. Goddard III, Angew. Chem. 1990, 102, 119-156: Angew. Chem. Int. Ed. Eng. 1990, 29, 138-175.

[8] I. F. G. A. Jansen. E. M. M. de Brabander-van den Berg. E. W. Meijer, Science 1994, 266, 1226-1229.

[9] C. J. Hawker, J. M. J. Fréchet, J. Am. Chem. Soc. 1990, 112, 7638-7642.

[10] Y. H. Liao, J. R. Moss, J. Chem. Soc. Chem. Commun. 1993, 1774-1777; G. R. Newkome, F. Cardullo, E. C. Constable, C. N. Moorefield. A. M. W. Cargill Thompson, ibid. 1993, $925-927$; M. F. Ottaviani, S. Bossmann, N. J. Turro, D. A. Tomalia, J. Am. Chem. Soc. 1994, 116, 661-671; A. Miedaner, C. J. Curits, R. M. Barkley, D. L. Dubois, Inorg. Chem. 1994, 33, 5483-5498; G. R. Newkome, R. Guther, C. N. Moorefield, F. Cardullo, L. Echegoyen, E. PerézCordero, H. Luftmann, Angew. Chem. 1995, 107, 2159-2162; Angew. Chem. Int. Ed. Engl. 1995, 34, 2023-2026.

[11] J. W. J. Knapen. A. W. van der Made, J. C. de Wilde, P. W. N. M. van Leeuwen, P. Wijkens, D. M. Grove, G. van Koten, Nature 1994, 372, 659-662.

[12] S. Campagna, G. Denti, S. Serroni, A. Juris, M. Venturi, V. Ricevuto, V. Balzani, Chem. Eur. J. 1995, 1, 211-221.
[13] S. Achar, R. J. Puddephat, Angew. Chem. 1994, 106, 895-897; Angew. Chem. Int. Ed. Engl. 1994, 33, 847-849.

[14] W. T. S. Huck, F. C. J. M. van Veggel, B. L. Kropman, D. H. A. Blank, E. G. Keim, M. M. A. Smithers, D. N. Reinhoudt, J. Am. Chem. Soc. 1995, 117, $8293-8294$.

[15] S. J. Loeb, G. K. H. Shimizu, J. Chem. Soc. Chem. Commun. 1993, 1395-1397.

[16] Die Koordination der Cyanogruppen ergibt sich aus der charakteristischen Verschiebung von $2252 \mathrm{~cm}^{-1}$ (freies $\mathrm{C} \equiv \mathrm{N}$ ) nach $2290 \mathrm{~cm}^{-1}:$ B. N. Storrhoff, H. C. Lewis, Coord. Chem. Rev. 1977, 23, 1-23.

[17] M. H. P. van Genderen, M. W. P. L. Baars, J. C. M. van Hest, E. M. M. de Brabander-van den Berg, E. W. Meijer, Recl. Trav. Chim. Pays-Bas 1994, 113. $573-574$

[18] Die Signale bei niedrigeren $m / z$-Werten im Spektrum von $\mathbf{G}_{2}$ entsprechen Spezies nach dem Verlust von einem, zwei oder drei Dendrimerbausteinen sowie von Anionen.

[19] Beispiele für den Verlust von Gegenionen: E. Leize, A. van Dorsselaer, R. Krämer, J.M. Lehn, J. Chem. Soc. Chem. Commun. 1993, 990-993; G. Hopfgarter, C. Piguet, J. D. Henion, A. F. Williams, Helv. Chim. Acta 1993, 76, $1759-1766$.

[20] Universal-Kraftfeld im Cerius ${ }^{2}$-Programmpaket: A. K. Rappé. C. J. Casewit, K. S. Colwell, W. A. Goddard III, W. M. Skiff, J. Am. Chem. Soc. 1992, 114, 10024-10035.

\section{Molekulare Kästen auf der Basis von Calix[4]aren-Doppelrosetten**}

Remko H. Vreekamp, John P. M. van Duynhoven, Martin Hubert, Willem Verboom und David N. Reinhoudt*

Dem Aufbau von nichtkovalent gebundenen Übermolekülen mit definierter Zusammensetzung gilt seit einigen Jahren wachsende Aufmerksamkeit. Die Erforschung der molekularen Selbstorganisation verspricht Informationen darüber, wie Wechselwirkungen zwischen Molekülfunktionen zu Einheiten mit neuen Formen und Funktionen führen. Diese können wie wie Kästen ${ }^{[1,2]}$, Kanäle ${ }^{[3]}$ oder komplexere dreidimensionale Netzwerke und andere diskrete Aggregate aufgebaut $\operatorname{sein}^{[4-6]}$. Die bislang beschriebenen, von nichtkovalenten Wechselwirkungen zusammengehaltenen molekularen Kästen weisen alle recht kleine Hohlräume auf, da sie durch Dimerisierung zweier Moleküle mit konkaver Struktur entstehen ${ }^{[2]}$.

Kürzlich wurden von uns Rezeptoren mit großen hydrophoben Hohlräumen durch kovalente Verknüpfung von Calix[4]arenen und Resorcin[4]arenen hergestellt ${ }^{[7]}$. Die Eigenschaften der erhaltenen Moleküle ermutigten uns, Calix[4]aren ${ }^{[8]}$ als Baustein zu verwenden, da es eine starre, konkave Oberfläche aufweist.

Wir beschreiben hier große, durch Selbstorganisation entstehende Kästen, die eine Doppelrosetten-Struktur aufweisen. Das Calix[4]aren-Gerüst ${ }^{[9]}$ dient als Abstandshalter zwischen den Rosetten, und es richtet die wasserstoffverbrückten Gruppen aus. So beträgt der Abstand zwischen den beiden Rosettenzentren CPK-Molekülmodellen ${ }^{[10]}$ zufolge $8.5 \AA$. Die Bildung der Rosette ist das Resultat der Wasserstoffbrücken zwischen Melamin-Einheiten und Barbitur- sowie Cyanursäure-Derivaten.

[*] Prof. Dr. Ir. D. N. Reinhoudt, Dr. Ir. R. H. Vreekamp, M. Hubert, Dr, W. Verboom

Laboratory of Organic Chemistry and MESA Research Institute

University of Twente

P.O. Box 217, NL-7500 AE Enschede (Niederlande)

Telefax: Int. $+53 / 4894645$

E-mail: orgchem

Dr. J. P. M. van Duynhoven

Laboratorium für Chemische Analyse, Universität Twente

[**] Wir danken der Akzo Nobel Central Research b.v. für die finanzielle Unterstützung. 
Diese Struktureinheit wurde von Whitesides et al. intensiv untersucht ${ }^{[11]}$, und auch Lehn et al. haben sie für Untersuchungen zur Selbstorganisation verwendet ${ }^{[12]}$.

Als Komponenten für die Doppelrosetten verwendeten wir drei verschiedene Calix[4]arene: das Dimelamin-Derivat 1 $\left(\mathrm{ClxM}_{2}\right)$, das am oberen Rand substituierte Isocyanursäure-Derivat 2 (ClxCA) und das am unteren Rand substituierte Isocyanursäure-Derivat 3 ( $t \mathrm{Bu}-\mathrm{ClxCA})^{[13]}$.

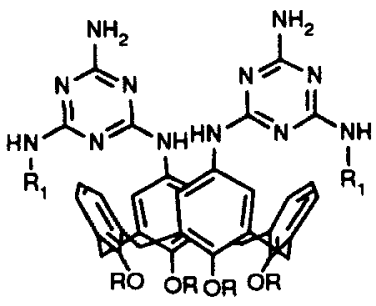

1

$\mathrm{R}=\mathrm{C}_{12} \mathrm{H}_{25}, \mathrm{R}_{1}=\mathrm{C}_{4} \mathrm{H}_{9}$

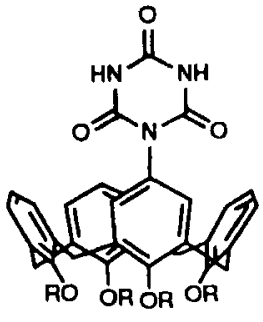

2

$R=\mathrm{C}_{12} \mathrm{H}_{2 \mathrm{~s}}$

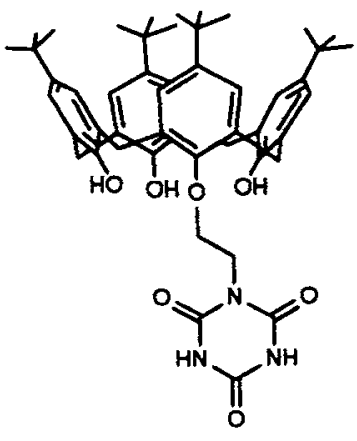

3

Eine Titration von 1 unter ${ }^{1} \mathrm{H}$-NMR-spektroskopischer Verfolgung mit Diethylbarbitursäure (BA) in $\mathrm{CDCl}_{3}(\mathrm{Abb}$. 1) ergab interessanterweise, daß schon bei geringer BA-Konzentration zwei Signale $(a, b)$ bei sehr tiefem Feld sichtbar werden. Diese Resonanzen ( $\delta=14.10$ und 13.32) ändern ihre Lage in Abhängigkeit vom Verhältnis 1:BA nicht und wurden von uns den wasserstoffverbrückten NH-Protonen von BA im 1-BA-Komplex zugeordnet. Erhöht man die Menge an BA, nimmt das Signal der $\mathrm{NH}_{2}$-Protonen von freiem 1 an Intensität $a b$, ohne $\mathrm{da} B$ sich die chemische Verschiebung ändert. Gleichzeitig treten zwei neue Signale bei $\delta=8.37$ und 7.43 hinzu (Signal c bzw. $\mathrm{d}$ in Abb. 1). Diese werden den beiden sekundären Amin-Protonen von $1 \mathrm{im}$ wasserstoffverbrückten Komplex zugeordnet. Spektren von Lösungen mit einem 1:BA-Verhältnis von 1:2 enthalten scharfe Signale, die auf das Fehlen von freiem 1 hinweisen. Das 1:2-Verhältnis ist mit dem Auftreten der Doppelrosetten-Struktur, die schematisch in Abbildung 2 gezeigt ist, in Einklang. Ein Verdünnen dieser Lösung erbrachte keine Veränderung im 'H-NMR-Spektrum, was darauf hindeutet, daß keine höher aggregierten Verbindungen auftreten. Werden mehr als zwei Äquivalente eingesetzt, sind die NH-Signale der freien Säure (x) neben den beiden der wasserstoffverbrückten im Spektrum enthalten, was auf einen auf der NMR-Zeitskala langsamen Austausch zwischen wasserstoffverbrückter und freier Säure hinweist ${ }^{[15]}$. Als Teil der Rosette weisen die beiden NHProtonen wegen der unsymmetrischen Substitution der Melamin-Einheiten eine unterschiedliche chemische Verschiebung auf. Für die beiden $\mathrm{NH}_{2}$-Protonen $(e, f)$ und die beiden Proto-

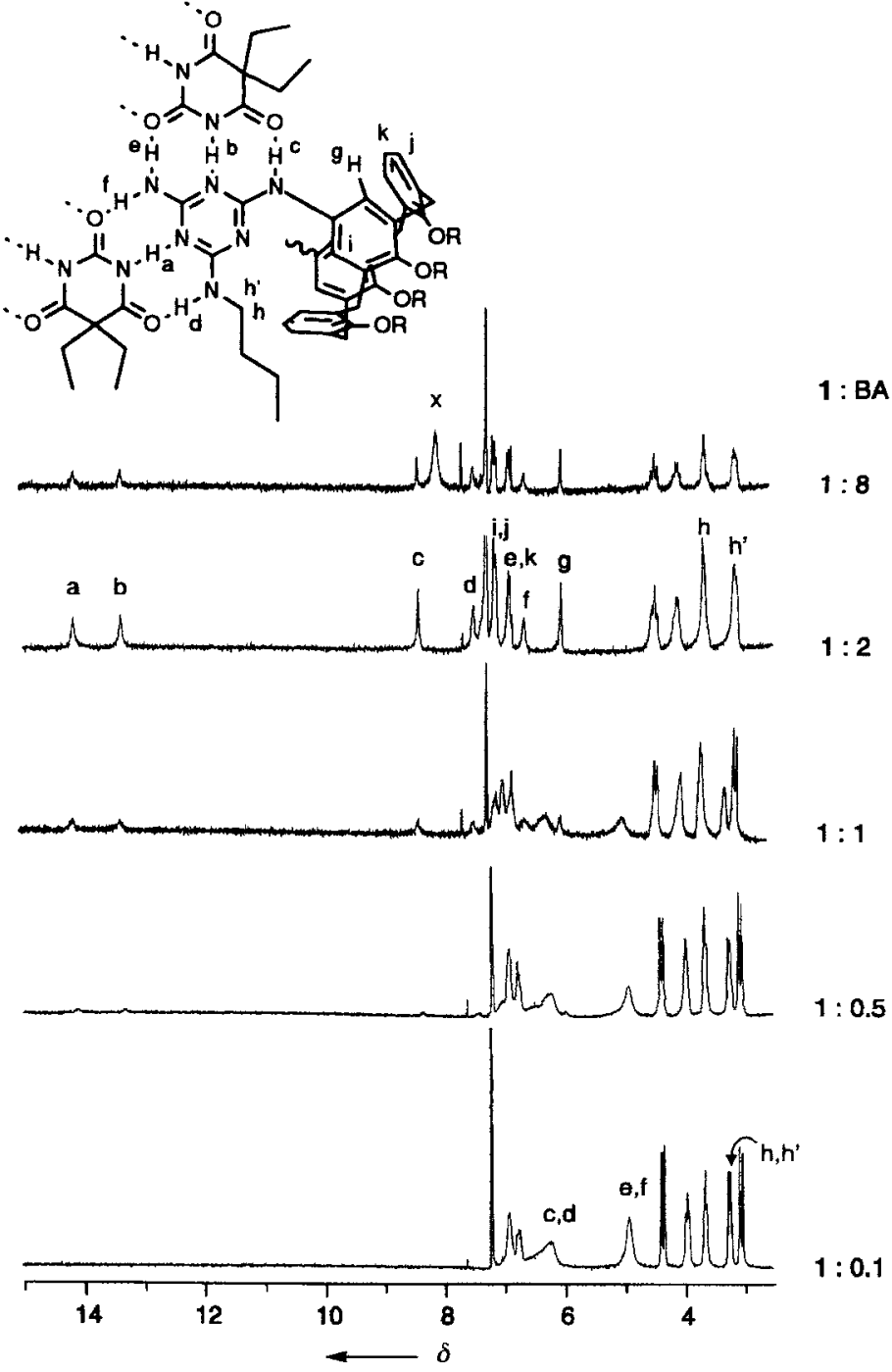

Abb. 1. ${ }^{1} \mathrm{H}$-NMR-Titration von 1 mit $\mathrm{BA}$ bei $400 \mathrm{MHz}$ bei Raumtemperatur. $10 \mathrm{~mm} \mathrm{CDCl}{ }_{3}$-Lösungen von 1 und $\mathrm{BA}$ wurden in verschiedenen Verhältnissen gemischt. Zur Kennzeichnung der Signale siehe Valenzstrichformel. $\mathrm{x}=$ Signal der freien Säure.

nen der $n$-Butyl-Seitenkette neben dem Stickstoffatom $\left(\mathrm{h}, \mathrm{h}^{\prime}\right)$ werden zwei Signale beobachtet, was auf Diastereotopizie hinweist $^{[16]}$.

Die Protonen des Melamin-substituierten aromatischen Rings von 1 ergeben zwei Signale, bei $\delta=7.15$ (i) sowie 6.03 (g). In freiem 1 findet man für diese Protonen breite Signale bei $\delta=7.05-6.70$ bzw. 6.65-6.05. Die Verschiebung bei $\delta=$ 6.03 ist im Einklang mit dem Auftreten einer verengten coneStruktur ${ }^{[17]}$, in der die beiden Melamin-Substituenten sich einander nähern ${ }^{[18]}$.

Eine Titration von 1 mit 2 brachte ähnliche Ergebnisse. Große Tieffeldverschiebungen werden für die NH-Protonen von 2 ( $\delta=15.43$ und 14.15) beobachtet, freie und wasserstoffverbrückte Verbindungen tauschen NMR-Spektren zufolge nicht aus, und ein 1:2-Verhältnis von 1:2 kann abgeleitet werden. Bei Verhältnissen unterhalb 1:2 (z.B. Überschuß von 1) treten nur Signale auf, die mit dem Vorliegen einer gesättigten Doppelrosetten-Struktur und freiem 1 in Einklang sind. Insbesondere bei tiefem Feld gibt es keine weiteren Signale, was bedeutet, daß keine Intermediate wie teilweise gefüllte Doppelrosetten oder lineare Aggregate gebildet werden. Dies deutet darauf hin, daß die Rosette, die in Abbildung 3 gezeigt ist, in 


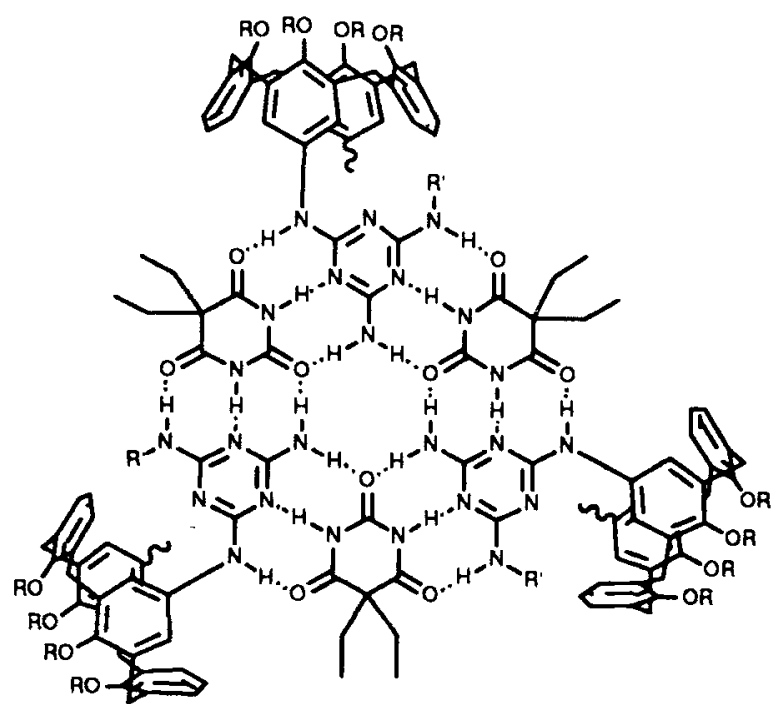

1・ BA

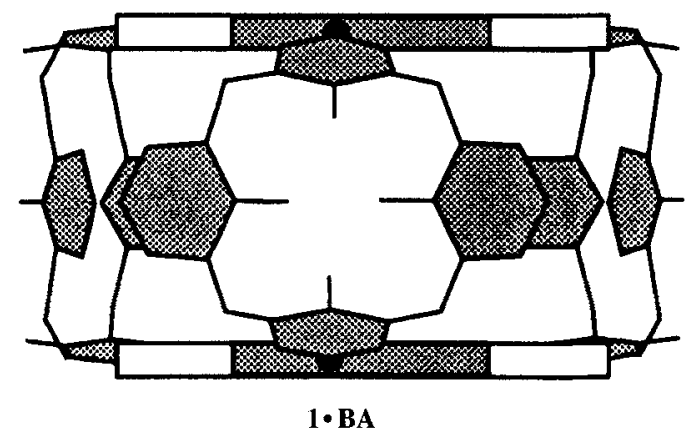

Abb. 2. Schematische Darstellung der postulierten Struktur der aus 1 und BA bestehenden Doppelrosette. Oben: Ansicht von oben (aus Gründen der Übersichtlichkeit ist nur der obere Teil einer der beiden Rosetten in der Ansicht von oben gezeigt). Unten: Seitenansicht.

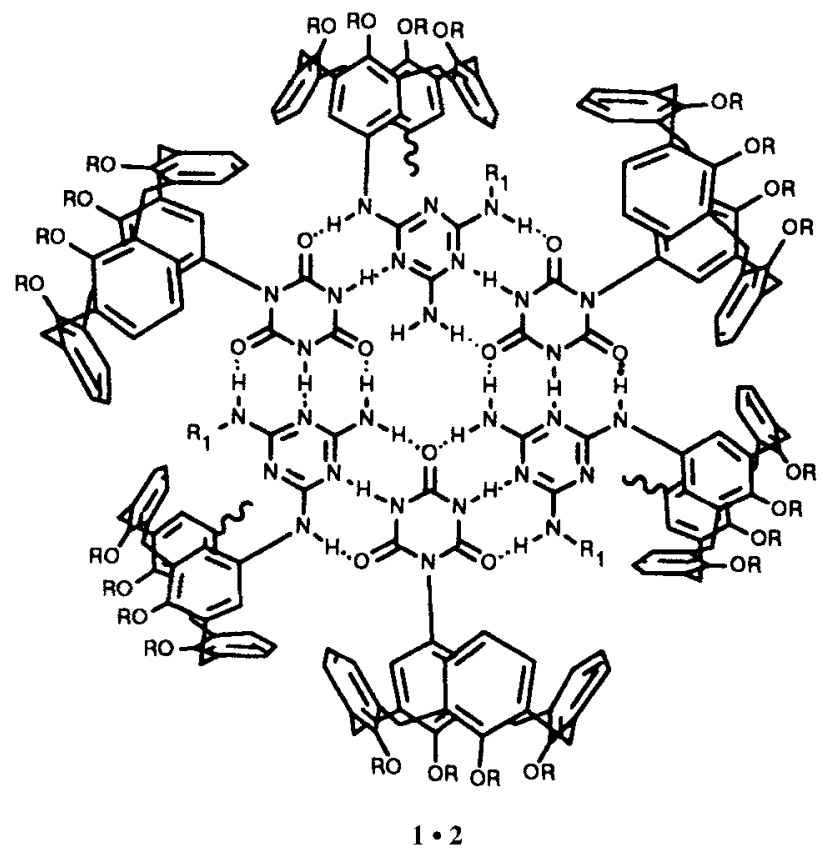

Abb. 3. Schematische Darstellung der postulierten Struktur der aus 1 und 2 bestehenden Doppelrosette (aus Gründen der Übersichtlichkeit ist nur der obere Teil einer der beiden Rosetten gezeigt). einem kooperativen Prozeß gebildet wird. Dabei werden während der Entstehung der einen Rosette die verbleibenden Einheiten geordnet, so daß die zweite Rosette bevorzugt gebildet wird.

Eine ${ }^{\prime} \mathrm{H}-\mathrm{NMR}$-Titration von 1 mit 3 brachte bis zu einem gewissen Grad ähnliche Ergebnisse wie die Titration von 1 mit BA. Die Signale der wasserstoffverbrückten NH-Protonen erscheinen bei etwas höherem Feld ( $\delta=15.17$ und 14.47). Bei einem 1:2-Verhältnis von $1: 3$ treten keine Signale von freiem 1 mehr auf ${ }^{[19]}$. Die Abstände zwischen den relevanten Protonen bei den drei verschiedenen Doppelrosetten wurden durch 2DNOESY-Experimente $(400 \mathrm{MHz})$ bestimmt, wobei die Anfangsgeschwindigkeitsnäherung verwendet wurde (Tabelle 1$)^{[20,21]}$. Die Abstände sind etwas größer als die, die mit CPK-Molekülmodellen und Molecular-Modeling-Rechnungen an ähnlichen wasserstoffverbrückten Struktureinheiten ermittelt wurden ${ }^{[22,23]}$, sie machen aber dennoch die vorgeschlagenen Rosetten-Strukturen plausibel.

Tabelle 1. Abstände von H-Atomen $[\AA ̊]$ in $\mathbf{1} \cdot \mathrm{BA}, \mathbf{1} \cdot \mathbf{2}$ und $\mathbf{1} \cdot \mathbf{3}$ [a].

\begin{tabular}{llll}
\hline H-A tome & $\mathbf{1} \cdot \mathrm{BA}$ & $\mathbf{1} \cdot \mathbf{2}$ & $\mathbf{1} \cdot \mathbf{3}$ \\
\hline $\mathrm{a}-\mathrm{d}$ & 2.8 & 2.5 & 2.7 \\
$\mathrm{a}-\mathrm{f}$ & 3.4 & 3.1 & 3.0 \\
$\mathrm{~b}-\mathrm{e}$ & 3.2 & 3.0 & 2.9 \\
$\mathrm{~b}-\mathrm{c}$ & 3.5 & 2.9 & 2.9 \\
$\mathrm{c}-\mathrm{g}$ & 3.2 & 2.9 & \\
\hline
\end{tabular}

[a] Die Abweichungen betragen $\pm 0.1 \AA$.

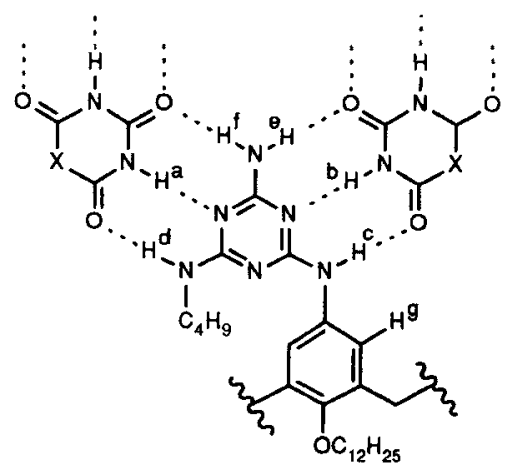

Die Ergebnisse weisen deutlich auf die Entstehung von Rosetten $^{[24]}$ hin. Ein weiterer Beweis hierfür läßt sich anhand des NOE-Aufbaus zwischen den Protonen der Methylenbrücken ableiten. Mit diesen Daten lassen sich die Kreuzrelaxationszeiten ermitteln, deren Größe und Vorzeichen in Beziehung zur Rotations-Korrelationszeit $\tau_{\mathrm{c}}$ stehen. Nimmt man ein zufälliges isotropes Taumeln eines sphärischen Moleküls oder Aggregats und die Abwesenheit einer inneren Bewegung an, läßt sich $\tau_{c}$ zum hydrodynamischen Volumen in Beziehung setzen ${ }^{[23,25,26]}$. Unter Anwendung der Anfangsgeschwindigkeitsnäherung wurden die Kreuzrelaxationszeiten für die Protonen der Methylenbrücken der drei Aggregate zusammen mit denjenigen für freies 3 und 1 bestimmt, und ausgehend von diesen Werten wurden die zugehörigen Werte für $\tau_{c}$ bestimmt. In der Regel steigen die $\tau_{c}$-Werte, was mit der Zunahme des erwarteten Molekulargewichts in Beziehung steht. Qualitativ besteht eine gute Korrelation mit den berechneten $\tau_{c}$-Werten (Abb. 4) ${ }^{[27]}$.

Die absoluten Abweichungen zwischen den berechneten und den experimentell bestimmten Werten beruhen wahrscheinlich auf unsicheren Annahmen hinsichtlich der Dichte und Form. Insbesondere bei den Annahmen über die Form besteht für die abgeflachten Doppelrosetten ein großer Unsicherheitsfaktor. Bekanntermaßen führt eine größere Elliptizität eines Moleküls 


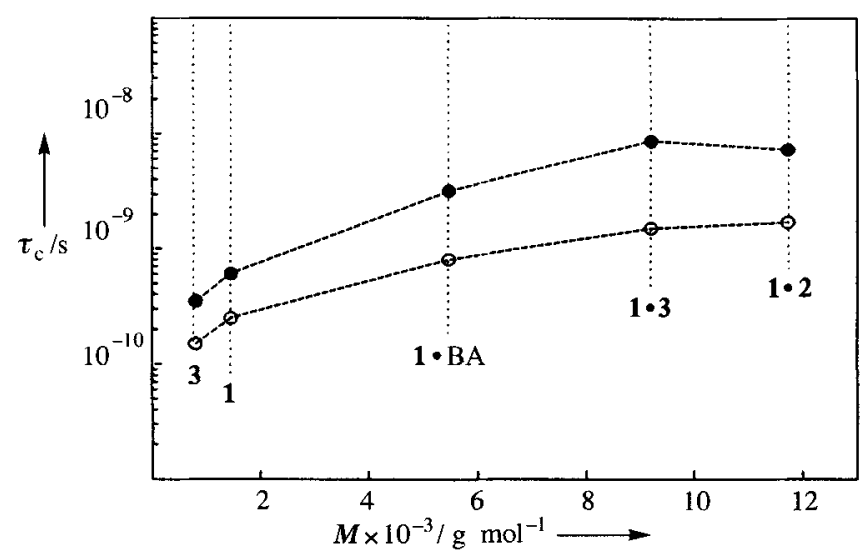

Abb. 4. Rotations-Korrelationszeiten $\tau_{\mathrm{e}}$, die aus den Kreuzrelaxationskonstanten als Funktion der Molekulargewichte der betreffenden Spezies bestimmt wurden: - = experimentell (400 MHz, $25 \mathrm{mM} \mathrm{CDCl}_{3}$.Lösungen); 0 = berechnet.

zu einer größeren effektiven Korrelationszeit, was mit einem scheinbar größeren Molekulargewicht in Einklang steht ${ }^{[28]}$.

Es gibt überzeugende Hinweise für die Bildung von Doppelrosetten aus den Ergebnissen von ${ }^{1} \mathrm{H}-\mathrm{NMR}$-Titrationen und aus den Abständen zwischen den an H-Brücken beteiligten Protonen, die durch NOE-Spektroskopie ermittelt wurden. Die Ergebnisse sind mit der erwarteten 1:2-Stöchiometrie im Einklang und legen nahe, daß es sich bei der Bildung der Doppelrosetten um einen hochgradig kooperativen Prozeß handelt. Zusätzlich sprechen Kreuzrelaxationsgeschwindigkeits-Messungen für die Bildung großer Einheiten, wie sich etwa aus dem Ansteigen der Rotationskorrelationszeiten bei der Bildung der Aggregate ergibt. Wir glauben, daß die Bestimmung der Kreuzrelaxationszeiten generell eine wertvolle Methode zur Charakterisierung supramolekularer Strukturen ist. Die zukünftige Arbeit konzentriert sich auf die Komplexierung von Gastmolekülen, die Kontrolle der Hohlraumgröße und die Größe der Öffnungen sowie die Einführung chemischer Funktionalitäten.

Eingegangen am 12. Dezember 1995 [Z 8638]

Stichworte: Calixarene $\cdot$ Selbstorganisation $\cdot$ Supramolekulare Chemie $\cdot$ Wasserstoffbrücken

[1] O. F. Schall, G. W. Gokel, J. Am. Chem. Soc. 1994, 116, 6089-6100.

[2] N. Branda, R. M. Grotzfeld, C. Valdés, J. Rebek, Jr., J. Am. Chem. Soc. 1995, $117,85-88$

[3] M. R. Ghadiri, J. R. Granja, L. K. Buehler, Nature, 1994, 369, 301-304

[4] S. J. Geib, C. Vicent, E. Fan, A. D. Hamilton, Angew. Chem. 1993, 105, 83-85; Angew. Chem. Int. Ed. Engl. 1993, 32, 119-121

[5] S. C. Zimmerman, B. F. Duerr, J. Org. Chem. 1992, 57, 2215-2217.

[6] J. L. Sessler, B. Wang, A. Harriman, J. Am. Chem. Soc. 1995, 117, 704-714

[7] a) P. Timmerman, W. Verboom, F. C. J. M. van Veggel, J. P. M. van Duynhoven, D. N. Reinhoudt, Angew. Chem. 1994, 106, 1313-1315; Angew. Chem. Int. Ed. Engl. 1994, 33, 1292-1295; b) P. Timmerman, K. G. A. Nierop, E. A. Brinks, W. Verboom, F. C. J. M. van Veggel, W. P. van Hoorn, D. N. Reinhoudt, Chem. Eur. J. 1995, 1, 124-131.

[8] Für eine aktuelle Übersicht über Calixarene siehe V. Böhmer, Angew. Chem 1995, 107, 785-818; Angew. Chem. Int. Ed. Engl. 1995, 34, 713-745.

[9] Einige über H-Brücken gebundene Aggregate, die auf Calix[4]arenen basieren sind beschrieben worden: a) J.-D. van Loon, R. G. Janssen, W. Verboom, D. N Reinhoudt, Tetrahedron Lett. 1992, 35, 5125-5128; b) K. Koh, K. Araki, S. Shinkai, ibid. 1994, 35, 8255-8258; c) A. Arduini, M. Fabbi, M. Mantovani, L. Mirone, A. Pochini, A. Secchi, R. Ungaro, J. Org. Chem. 1995, 69, $1454-$ 1457.

[10] Dies ist wesentlich mehr als wenn ein einfacher meta-Xylyl-Abstandshalter benutzt würde (1-2 ̊) : J. P. Mathias, E. E. Simanek, G. M. Whitesides, J. Am. Chem. Soc. 1994, 116, 4326-4340.

[11] Übersicht: G. M. Whitesides, E. E. Simanek, J. P. Mathias, C. T. Seto, D. N Chin, M. Mammen, D. M. Gordon, Acc. Chem. Res. 1995, 28, 37-44
[12] K. C. Russell, E. Leize, A. van Dorsselaer, J-M. Lehn, Angew. Chem. 1995. 107, 244-248; Angew. Chem. Int. Ed. Engl. 1995, 34, 209-213.

[13] 1 wurde in fünf Schritten ausgehend vom de-tert-butyliertem Calix[4]aren in einer Gesamtausbeute von $19 \%$ erhalten. Die Reaktionen umfassen eine Alkylierung am unteren Rand, eine selektive Nitrierung des oberen Rands [14]. gefolgt von einer Reduktion zum Diamin, die Reaktion mit Cyanursäurechlorid, Ammoniak und $n$-Butylamin. Die ersten vier Schritte der Synthese von 2 sind denen zur Synthese von 1 sehr ähnlich. Mononitriertes de-tert-butyliertes Calix[4]aren wurde zum Monoamin reduziert. Anschließende Reaktion mit Nitrobiuret lieferte das Biuret-Derivat, das mit Diethylcarbonat in die Isocyanursäure urngewandelt wurde; reines 2 wurde in einer Gesamtausbeute von $22 \%$ erhalten. Die Herstellung von 3 begann mit der Monoalkylierung von p-tertButylcalix[4]aren mit 2-Brom-ethylphthalimid. Das resultierende PhihalimidDerivat wurde quantitativ zum Monoamin reduziert, welches dann mit Nitrobiuret umgesetzt wurde. Nach der Reaktion mit Diethylcarbonat wurde reines 3 erhalten. Alle neuen Verbindungen wurden mit ${ }^{1} \mathrm{H}-\mathrm{NMR}$ - und ${ }^{13} \mathrm{C}-\mathrm{NMR}$ Spektroskopie, FAB-Massenspektrometrie und Elementaranalyse charakterisiert.

[14] J.-D. van Loon, J. F. Heida, W. Verboom, D. N. Reinhoudt, Red. Trav. Chim. Pays-Bas 1992, 111, 353-359.

[15] Aus der großen Differenz der chemischen Verschiebung zwischen freiem und komplexiertem BA (Abb. 1) läßt sich als obere Grenze für die DissoziationsGeschwindigkeitskonstante ein Wert von ca. $2000 \mathrm{~s}^{-1}$ abschätzen. Nimmı man an, da $\beta$ die Geschwindigkeit der Bildungsreaktion diffusionsbestimmt ist, kann die Geschwindigkeitskonstante auf $\geq 10^{5} \mathrm{M}^{-1}$ abgeschätzt werden.

[16] Für die Zuordnung der Signale des 1-BA-Komplexes und der Signale der übrigen in dieser Arbeit besprochenen Anordnungen wurde die NOE-, ROE- und J-Korrelationsspektroskopie verwendet.

[17] Verengt bedeutet, da $B$ zwei gegenüberliegende aromatische Ringe fast parallel zueinander liegen, während die anderen beiden eine abgeflachte Position einnehmen.

[18] Die Rosette ist auch in Acetonitril und Toluol stabil. Der Zusatz von mehr als $10 \%$ DMSO oder $\mathrm{MeOH}$ zu einer $\mathrm{CDCl}_{3}$-Lösung führte zu langsamer Zersetzung.

[19] In diesem Fall werden - bei Einsatz von mehr als zwei Äquivalenten 3 - als Folge von Wasserstoffbrückenbindungen zwischen freiem 3 und den HydroxyGruppen von 3 innerhalb des Aggregats wahrscheinlich größere Aggregate gebildet; dies läßt sich aus dem Auftreten neuer Signale im ${ }^{1} \mathrm{H}-\mathrm{NMR}-\mathrm{Spek}$ trum ableiten.

[20] R. R. Ernst, G. Bodenhausen, A. Wokaun in Principles of Nuclear Magnetic Resonance in One and Two Dimensions; International Series of Monographs on Chemistry, Vol. 14 (Hrsg.: R. Breslow, J. B. Goodenough, J. Halpern, D. Rowlingson), Clarendon, Oxford, 1987, S. 490-538.

[21] Ein Wert von $1.79 \AA$ für den Abstand zwischen den axialen und äquatorialen Protonen der Methylenbrücken wurde als Berechnungsgrundlage verwendet.

[22] J. Pranata, S. G. Wierschke, W. L. Jorgensen, J. Am. Chem. Soc. 1991, 113, $2810-2819$

[23] K. Wüttrich, NMR of Proteins and Nucleic Acids, Wiley, New York, 1986

[24] Die 1-BA- und 1-2-Rosetten-Bildung wurde auch mit Hilfe von ElectrosprayMS bestätigt, wobei Werte von $m / z$ von 5770 (berechnet für $M+7 \mathrm{MeCN}$ 5768) bzw. 11727 (berechnet für $M+3 \mathrm{H} 11$ 727) erhalten wurden. Im Fall des 1-2-Moleküls ergaben VPO-Messungen in $\mathrm{CHCl}_{3}$ bei $29^{\circ} \mathrm{C}$ ein Molekulargewicht von $10.0 \pm 1.8 \times 10^{3} \mathrm{~g} \mathrm{~mol}^{-1}$ (berechnet 9199).

[25] D. C. Fry, S. A. Kuby, A. S. Mildvan, Biochemistry 1985, 24, 4680-4693.

[26] Das hydrodynamische Volumen ist mit der Molmasse über die Dichte $\varrho$ verknüpft. Für die Bestimmung von $\tau_{\mathrm{c}}$ wird Gleichung (a) verwendet.

$\left.\sigma_{12}=\frac{1}{10}\left(\frac{\mu_{0}}{4 \pi}\right)\right)^{2} \frac{\gamma_{\mathrm{H}}^{4} h^{2}}{r^{6}}\left\{\frac{6 \tau_{\mathrm{c}}}{1+4 \omega^{2} \tau_{\mathrm{c}}^{2}}-\tau_{\mathrm{c}}\right\}$

$\mu_{0}=$ Dielektrizitätskonstante, $\gamma=$ gyromagnetische Konstante, $r=$ Abstand zwischen den Atomen 1 und 2, $\omega=$ Larmor-Frequenz.

Diese Gleichung drückt die Beziehung zwischen $\tau_{\mathrm{c}}$ und der KreuzrelaxationsKonstanten für ein Zwej-Spin-System unter Beteiligung der Atome 1 und 2, $\sigma_{12}$, aus, wobei eine isotrope Taumelbewegung und reine Dipol-Dipol-Relaxation angenommen wird

[27] Für diese Berechnungen wurden folgende Werte in die Stokes-Gleichung eingesetzt: $T=303 \mathrm{~K} ; \eta_{0}=0.514 \mathrm{cP}$.

[28] a) T. L. James, G. B. Matson, I. D. Kuntz, J. Am. Chem. Soc. 1978, 100, 35903594; b) C. F. Morgan, T. Schleich, G. H. Caines, D. Michael, Biopolymers 1990, $29,469-480$ 